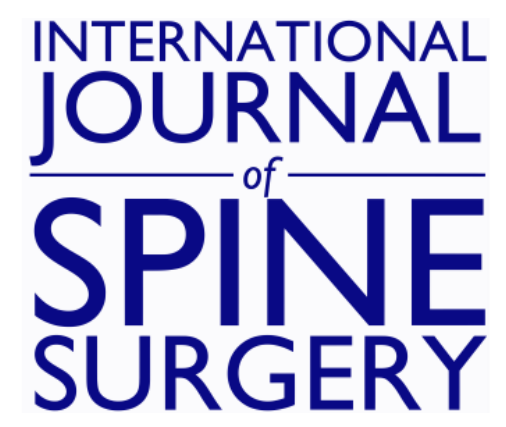

\title{
Ninety-day readmissions after degenerative cervical spine surgery: A single-center administrative database study
}

Chibuikem Akamnonu, Thomas Cheriyan, Jeffrey A. Goldstein, Thomas J. Errico and John A. Bendo

Int J Spine Surg 2015, 9 ()

doi: https://doi.org/10.14444/2019

http://ijssurgery.com/content/9/19

This information is current as of April 26, 2023.

Email Alerts Receive free email-alerts when new articles cite this article. Sign up at:

http://ijssurgery.com/alerts

The International Journal of Shing Surgerih 2397 Waterbury Circle, Suite 1,

Aurora, IL 60504, Phone: +1-630-375-1432

(C) 2015 ISASS. All Rights Reserved. 


\section{Ninety-day readmissions after degenerative cervical spine surgery: A single-center administrative database study}

Chibuikem Akamnonu MD, ${ }^{1}$ Thomas Cheriyan MD, ${ }^{1}$ Jeffrey A. Goldstein MD, ${ }^{1}$ Thomas J. Errico MD, ${ }^{1}$ John A. Bendo MD

${ }^{1}$ Division of Spine Surgery, Hospital for Joint Diseases, New York Langone Medical Center, New York

\section{Abstract}

\section{Background}

Unplanned hospital readmissions result in significant clinical and financial burdens to patients and the healthcare system. Readmission rates and causes have been investigated using large administrative databases which have certain limitations in data reporting and coding. The objective of this study was to provide a description of 90 day post-discharge readmissions following surgery for common degenerative cervical spine pathologies at a largevolume tertiary hospital. The study also compared the readmission rates of patients who underwent anterior- and posterior-approach procedures.

\section{Methods}

The administrative records from a single-center, high-volume tertiary institution were queried using ICD-9 codes for common cervical pathology over a three year period to determine the rate and causes of readmissions within the 90 days following the index surgery.

\section{Results}

A total of 768 patients underwent degenerative cervical spine surgery during the three year study period. Within 90 days of discharge, 24 (3.13\%) patients were readmitted; 16 (2.06\%) readmissions were planned for lumbar surgery; 8 (1.04\%) readmissions were unplanned. 640 patients underwent procedures involving an anterior approach and 128 patients underwent procedures involving a posterior approach. There were 14 (2.17\%) planned readmissions in the anterior group and $2(1.5 \%)$ in the posterior group. The unplanned readmission rate was $0.63 \%$ (4 patients) and 3.13\% (4 patients) in the anterior and posterior groups, respectively. ( $\mathrm{p}=0.0343)$.

\section{Conclusion}

The 90 day post-discharge unplanned readmission rate that followed elective degenerative cervical spine surgery was $1.04 \%$. The unplanned readmission rate associated with posterior-approach procedures (3.13\%) was significantly higher than that of anterior-approach procedures $(0.63 \%)$.

Level of evidence: IV

KEYWORDS: READMISSION, CERVICAL, DEGENERATIVE SURGERY

VOLUME 9 ARTICLE 19 DOI: 10.14444/2019

\section{Introduction}

Hospital readmissions following surgery have major clinical and financial implications, especially in the fast evolving environment of the US healthcare system. Jencks et al. reported that the estimated annual cost from 30 day post-discharge hospital readmissions is approximately $\$ 17$ billion. ${ }^{1}$ Well-designed interventions have successfully reduced avoidable readmission rates by as much as $45 \% .^{2-5}$ Data regarding readmission causes and rates in spine surgery have been investigated using large billing administrative databases like the National Inpatient Sample and Medicare. ${ }^{6,7}$ However, these databases have inherent limitations such as diagnosis under-reporting, coding errors and lack of clinical detail. Further compounding the issue are the myriad, diverse factors that influence readmission rates and make it especially challenging to standardize an "acceptable" readmission rate. For example, geographic variations have been shown to impact readmission rates, possibly due to a threshold effect of hospital-bed availability. ${ }^{8}$ 
The use of single-center hospital administrative billing records to analyze early readmission rates and causes may mitigate some of the limitations of large databases. The objective of this study was to determine the 90 day readmission rate and causes after elective degenerative cervical surgery at an urban single-center tertiary institution. The study also aimed to compare readmission rates between anterior and posterior cervical approaches in the setting of degenerative cervical spine surgery.

\section{Material and Methods}

After institutional review board approval was obtained, administrative data from 2011 through 2014 was queried to obtain 90 day readmissions after elective degenerative spine surgery. The study was conducted at a 220-bed tertiary care orthopedic specialty institution with 24 fellowship-trained spine surgeons. International Classification of Disease, Ninth Revision Clinical Modification (ICD-9-CM) codes were used to identify select cervical spine diagnosis and cervical spine procedures. Patients were identified using the following inclusion criteria:

\section{Over 18 years of age;}

2. patients with primary diagnostic codes: 721.0 (cervical spondylosis without myelopathy), 721.1 (cervical spondylosis with myelopathy), 722.0 (displacement of cervical intervertebral disc without myelopathy), 722.4 (degeneration of cervical intervertebral disc), 722.71 (cervical intervertebral disc disorder,723.0 (cervical spinal stenosis), 723.4 (Brachial neuritis or radiculitis NOS), 996.67 (infection or inflammation reaction due to other internal orthopaedic device implant and graft), 998.59 (other post operative infection); and

3. patients with primary surgical procedure identified by ICD-9CM codes: 81.02 (other cervical fusion anterior/anterior), 81.03 (other cervical fusion posterior/posterior), 3.09 (cervical canal exploration NEC), 81.01 (atlas-axis fusion), 84.62 (cervical disc replacement).

Data on patient demographics, length of stay, payer, severity of disease as measured by the All Patient Refined-Diagnosis Related Groups (APR-DRG), and discharge disposition was obtained from administra- tive records.

All surgical procedures were divided into an anterior group and a posterior group based on the approach that was utilized during surgery. In this study, the anterior approach group was comprised exclusively of patients who underwent anterior cervical decompression/corpectomy with fusion and cervical total disc replacements procedures. The posterior approach group included patients who underwent cervical foraminotomy, laminoplasty and laminectomy/ fusions. Any surgeries that were conducted solely for the correction of primary cervical deformity were excluded from the study.

Using our electronic medical record, the chart from each readmission was further reviewed. Readmissions were grouped into two categories: planned and unplanned readmissions. "Planned readmission" was defined as a staged or rescheduled procedure including planned procedures involving a different anatomical region. "Unplanned readmissions" included readmissions due to either surgical or nonsurgical complications.

\section{Statistical Analysis}

Continuous data was reported as mean, standard deviation and range. Frequency distribution was used to describe categorical variables. Unpaired t-test, chisquare or Fishers exact test were used to compare variables between the anterior approach and posterior approach groups.

\section{Source of Funding}

There was no external source of funding for this study.

\section{Results}

A total of 768 patients were identified during the study period; 416 (54.2\%) were male and 352 (45.8\%) were female. The mean length of stay was $1.89 \pm 1.59$ days (range: 0-20). Distribution frequency of diagnosis, discharge disposition, surgical procedure, surgical approach, APR-DRG severity and payer in the patient cohort are described in Table 1 . There were 24 readmissions, of which 16 were planned and 8 were unplanned. Fifteen of the planned readmissions 
were indicated for surgical management of concomitant lumbar pathology and one was an anterior/posterior cervical surgery that was staged two weeks apart. Seven out of the eight unplanned readmissions were directly related to the index surgical procedure. The cause of unplanned readmissions is detailed in Table 2. There were three revisions for persistent neurological symptoms. One of these three revisions followed a C4-C7 ACDF for which a multi-level laminectomy/fusion was performed; another revision followed a C3-C7 laminoplasty for which a one-level ACDF was performed; the final revision followed a posterior laminectomy/fusion with postoperative radiculopathy in setting of new foraminal stenosis for which an ACDF was performed. Two unplanned readmissions were due to infection; one followed an $\mathrm{ACDF}$ and the other followed a trans-oral dens resection/atlas-axis posterior fusion. One patient had an unplanned readmission for persistent wound drainage following C6-C7 and C7-T1 unilateral foraminotomy for which irrigation and debridement was performed. However, it is important to note that this patient presented no evidence of infection during the initial hospital stay for index surgery and all cultures were negative. One patient was readmitted in connection with a fall. Lastly, one patient was readmitted for workup for a new spine metastasis diagnosis.

Among the 768 surgeries, 640 (83\%) surgeries involved an anterior approach and 128 (17\%) involved a posterior approach (Table 3 ). The mean age of patients in the posterior approach group was higher (60.7 \pm 11 years) when compared to the anterior approach group $(49.1 \pm 10.4$ years $)(\mathrm{p}<0.0001)$. There were $343(53.6 \%)$ and $73(57 \%)$ males in the anterior and posterior groups, respectively $(\mathrm{p}=0.50)$. The mean length of stay was $1.89 \pm 1.59$ days in the anterior group compared to $3.41 \pm 1.8$ days in the posterior group $(\mathrm{p}<0.0001)$. Distribution frequency of diagnosis, discharge disposition, surgical procedure, APRDRG severity and payer in the patient cohort is described in Table 3. Of the planned readmissions, 14 were done after $\mathrm{ACDF}$ and two followed posterior cervical fusion. There were four unplanned readmissions in each group; readmission rates were $0.63 \%$ and $3.13 \%$ in the anterior and posterior groups, respectively ( $\mathrm{p}=0.0343)$.

\section{Discussion}

The 90-day unplanned readmission rate following elective degenerative cervical spine surgery at our center was $1.04 \%$. The unplanned readmission rate

\begin{tabular}{|c|c|c|}
\hline & & $\begin{array}{r}\text { Mean/ } \\
\text { Frequency }\end{array}$ \\
\hline Age & & $\begin{array}{r}51.0 \pm 11.3 \\
\text { years }(22-89)\end{array}$ \\
\hline \multirow{3}{*}{ Gender } & Male & $416(54.2)$ \\
\hline & Female & $352(45.8)$ \\
\hline & Length of Stay & $\begin{array}{r}1.9 \pm 1.6 \text { days } \\
(0-20)\end{array}$ \\
\hline \multirow{7}{*}{ Diagnosis } & Brachial neuritis or radiculitis NOS & $14(1.82)$ \\
\hline & Cervical spondylosis with myelopathy & $177(23.05)$ \\
\hline & Cervical spondylosis without myelopathy & $54(7.03)$ \\
\hline & $\begin{array}{l}\text { Displacement of cervical intervertebral } \\
\text { disc without myelopathy }\end{array}$ & $367(47.79)$ \\
\hline & $\begin{array}{l}\text { Degeneration of cervical intervertebral } \\
\text { disc }\end{array}$ & $28(3.65)$ \\
\hline & cervical intervertebral disc disorder & $94(12.24)$ \\
\hline & cervical spinal stenosis & $34(4.43)$ \\
\hline \multirow{2}{*}{ Approach } & Anterior & $640(83)$ \\
\hline & Posterior & $128(17)$ \\
\hline \multirow{6}{*}{ Disposition } & Home / Self care & $607(79.03)$ \\
\hline & Home /Health care service & $128(16.67)$ \\
\hline & Rehabilitation Facility & $16(2.08)$ \\
\hline & Nursing Facility & $11(1.43)$ \\
\hline & Short-term hospital/ Health care facility & $5(0.65)$ \\
\hline & Expired & $1(0.13)$ \\
\hline \multirow{5}{*}{ Procedures } & Anterior fusion & $626(81.51)$ \\
\hline & Posterior fusion & $79(10.29)$ \\
\hline & Spinal canal exploration & $42(5.47)$ \\
\hline & Cervical disc replacement & $14(1.82)$ \\
\hline & Atlantoaxial fusion & $7(0.91)$ \\
\hline \multirow{3}{*}{$\begin{array}{l}\text { APR-DRG } \\
\text { disposition }\end{array}$} & One & $485(63.48)$ \\
\hline & Two & $259(33.90)$ \\
\hline & Three & $20(2.62)$ \\
\hline \multirow{3}{*}{ Payer } & Private & $526(67.8)$ \\
\hline & Medicare & $102(13.1)$ \\
\hline & Workers comp & 148 (19.1) \\
\hline
\end{tabular}

Table 2. Causes of unplanned readmissions.
\begin{tabular}{|l|r|}
\hline Cause & Number \\
\hline $\begin{array}{l}\text { Persistent neurological symptoms: } \\
\text { Revision surgery }\end{array}$ & 3 \\
\hline Infection & 2 \\
\hline Persistent wound drainage & 1 \\
\hline Postoperative weakness/ fall & 1 \\
\hline Workup for new diagnosis of spine metastasis & 1 \\
\hline
\end{tabular}


following posterior-approach procedures was higher than that of anterior-approach procedures (3.13\% versus $0.63 \%)$.

The determination of "higher than expected" readmission rates following surgical procedures has become an area of increasing concern. Our study aimed to report unplanned readmission rates for degenerative cervical spine procedures at a single center. The study has advantages over national database studies ${ }^{9,10}$ in that it provides more clinical detail and minimizes reporting errors. The 90 day readmission rate was based on the immediate post-operative period in the Medicare bundled payment plans. It is important to note that infections and wound complications - which constitute the majority of spine surgery complications - are less likely to present beyond this time period. . Schairer reported a 90 day unplanned readmission rate of $12.3 \%$ in patients who underwent deformity surgery in his study. ${ }^{11} \mathrm{McCor}-$ mack reported 30 day unplanned readmission rates of 4.3\% for anterior cervical spine fusion patients and $5.9 \%$ for posterior cervical fusion patients from 2007 through $2009 .{ }^{7}$ His study is of particular interest because it took place at the same institution as ours, yet it reported higher readmission rates than we observed from 2011 through 2014. These disparate findings can be explained, at least in part, by changes in institution protocol that included application of intrawound vancomycin powder in posterior cervical surgeries, (b) routine nasal swabbing for identification of Staphylococcus carriers in order to optimize antibiotic prophylaxis and (c) standardization of dressing protocol.

Lovecchio et al. ${ }^{9}$ reported a 30 day readmission rate of $2.5 \%$ among the 2320 patients who underwent ACDF from the National Quality Improvement

Table 3. Approach-based distribution of patient cohort.

\begin{tabular}{|c|c|c|c|c|}
\hline & & Anterior & Posterior & $P$ value \\
\hline Age & & $49.1 \pm 10.3$ years $(22-81)$ & $60.7 \pm 11.1$ years $(38-89)$ & $<0.0001$ \\
\hline \multirow{2}{*}{ Gender } & Male & $343(53.6)$ & $73(57.0)$ & \multirow{2}{*}{0.212} \\
\hline & Female & $297(46.4)$ & $55(43.0)$ & \\
\hline Length of Stay & & $1.9 \pm 1.6$ days $(1-20)$ & $3.4 \pm 1.8$ days $(0-10)$ & $<0.0001$ \\
\hline \multirow{7}{*}{ Diagnosis } & Brachial neuritis or radiculitis NOS & $12(1.9)$ & $2(1.6)$ & \multirow{7}{*}{$<0.0001$} \\
\hline & Cervical spondylosis with myelopathy & $99(15.5)$ & $78(61.0)$ & \\
\hline & Cervical spondylosis without myelopathy & $43(6.7)$ & $11(8.6)$ & \\
\hline & Displacement of cervical intervertebral disc without myelopathy & $357(55.8)$ & $10(7.8)$ & \\
\hline & Degeneration of cervical intervertebral disc & $24(3.8)$ & $4(3.1)$ & \\
\hline & Cervical intervertebral disc disorder & $85(13.3)$ & $9(7.0)$ & \\
\hline & Cervical spinal stenosis & $20(3.1)$ & $14(11.0)$ & \\
\hline \multirow{2}{*}{ Approach } & Anterior & 640 & $\mathrm{~N} / \mathrm{A}$ & \multirow{2}{*}{$\mathrm{N} / \mathrm{A}$} \\
\hline & Posterior & N/A & 128 & \\
\hline \multirow{6}{*}{ Disposition } & Home / Self care & $556(86.9)$ & $51(39.8)$ & \multirow{6}{*}{$<0.0001$} \\
\hline & Home /Health care service & $67(10.4)$ & $61(47.7)$ & \\
\hline & Rehabilitation Facility & $6(0.9)$ & $10(7.8)$ & \\
\hline & Nursing Facility & $5(0.8)$ & $6(4.7)$ & \\
\hline & Short-term hospital/ Health care facility & $4(0.6)$ & $0(0)$ & \\
\hline & Expired & $1(0.13)$ & $0(0)$ & \\
\hline \multirow{5}{*}{ Procedures } & Anterior fusion & $626(97.8)$ & 0 & \multirow{5}{*}{$<0.0001$} \\
\hline & Posterior fusion & 0 & $79(61.7)$ & \\
\hline & Spinal canal exploration & 0 & 439 & \\
\hline & Cervical disc replacement & $14(2.2)$ & $0(0)$ & \\
\hline & Atlantoaxial fusion & $0(0)$ & $7(5.5)$ & \\
\hline \multirow{3}{*}{ APR-DRG disposition } & One & $444(69.8)$ & $41(32.0)$ & \multirow{3}{*}{$<0.0001$} \\
\hline & Two & $179(28.1)$ & $80(62.5)$ & \\
\hline & Three & $13(2.0)$ & $7(5.5)$ & \\
\hline \multirow{3}{*}{ Payer } & Private & 439 (68.6) & $84(65.6)$ & \multirow{3}{*}{$<0.0001$} \\
\hline & Medicare & $60(9.3)$ & $39(30.5)$ & \\
\hline & Workers comp & $141(22.0)$ & $5(3.9)$ & \\
\hline
\end{tabular}

APR-DRG: All Patient Related-Diagnosis Related Groups. Values in parenthesis denote percentage or range depending if categorical or continuous variables, respectively.

Downloaded from http://ijssurgery.com/ by guest on April 26, 2023 
Database. Pulmonary complications, wound complications and urinary tract infections were the most common complications in their cohort of readmitted patients. Older age and perioperative factors such as hypertension and diabetes were also associated with high readmission rates. There were $3(0.48 \%)$ readmissions among the 626 patients who underwent an anterior fusion in our patient cohort. Wang et al. ${ }^{12}$ used a patient sample from Medicare beneficiaries and reported a 30 day readmission rate following cervical surgery of $7.9 \%$. In their cohort, the predictors for readmissions following cervical surgery were older age, dual eligibility for Medicare/Medicaid, greater co-morbidity, male sex, diagnosis of myelopathy, and a posterior or combined anterior/posterior approach.

King et al. ${ }^{6}$ and McCormack et al. ${ }^{7}$ have reported higher readmission rates after posterior approach surgery when compared to anterior approach. This is consistent with findings in our cohort of patients. Also, the average length of hospital stay was 1.9 days with a statistically difference between anterior versus posterior based procedures (1.6 versus 3.4 days respectively, $\mathrm{p}<0.0001)$. However, it is possible that the unplanned readmission rate in the posterior approach group might be higher than that of the anterior group because of a difference in patient ages and diagnoses as opposed to surgical approach choice per se.

Of the 768 patients in this study, 523 had private insurance, 146 had workers compensation and 99 had Medicare. Of those who had unplanned readmissions, there was a statistically significant difference $(\mathrm{p}=0.004)$ between the groups. Two $(0.38 \%)$ private insurance patients had unplanned readmissions; 2 (1.37\%) workers compensation patients had unplanned readmissions; 4 (4.04\%) Medicare patients had unplanned readmissions. This can be explained, at least in part, by the fact that the Medicare group contained the highest percentage of patients (5.2\%) who fell within the most severe subgroup of APRDRG; by contrast, the same was true of only $2.7 \%$ of those with private insurance and $.7 \%$ of those with workers compensation.

We recognize that there were several limitations of this study. Due to the low number of unplanned readmissions, risk factors associated with readmission could not be evaluated. Another limitation of this study is the possible underreporting of unplanned readmission rates, as patients might have sought treatment at different institutions for complications. This is especially true for non-surgical complications, and these data were not captured in this study.

\section{References}

1. Jencks SF, Williams MV, Coleman EA. Rehospitalizations among patients in the Medicare fee-forservice program. $N E n g l J$ Med. Apr 2 2009;360(14):1418-1428.

2. Coleman EA, Parry C, Chalmers S, Min SJ. The care transitions intervention: results of a randomized controlled trial. Arch Intern Med. Sep 25 2006;166(17):1822-1828.

3. Naylor MD, Brooten D, Campbell R, et al. Comprehensive discharge planning and home follow-up of hospitalized elders: a randomized clinical trial. $J A$ MA. Feb 17 1999;281(7):613-620.

4. Jack BW, Chetty VK, Anthony D, et al. A reengineered hospital discharge program to decrease rehospitalization: a randomized trial. Ann Intern Med. Feb 3 2009;150(3):178-187.

5. Mor V, Besdine RW. Policy options to improve discharge planning and reduce rehospitalization. $J A$ MA. Jan 19 2011;305(3):302-303.

6. King JT, Jr., Abbed KM, Gould GC, Benzel EC, Ghogawala Z. Cervical spine reoperation rates and hospital resource utilization after initial surgery for degenerative cervical spine disease in 12,338 patients in Washington State. Neurosurgery. Dec 2009;65(6):1011-1022; discussion 1022-1013.

7. McCormack RA, Hunter T, Ramos N, Michels $\mathrm{R}$, Hutzler L, Bosco JA. An analysis of causes of readmission after spine surgery. Spine (Phila $\mathrm{Pa}$ 1976). Jun 15 2012;37(14):1260-1266.

8. Fisher ES, Wennberg JE, Stukel TA, Sharp SM. Hospital readmission rates for cohorts of Medicare beneficiaries in Boston and New Haven. NEngl J Med. Oct 13 1994;331(15):989-995.

9. Lovecchio F, Hsu WK, Smith TR, Cybulski G, Kim B, Kim JY. Predictors of thirty-day readmission

Downloaded from http://ijssurgery.com/ by guest on April 26, 2023 
after anterior cervical fusion. Spine (Phila Pa 1976). Jan 15 2014;39(2):127-133.

10. Modhia U, Takemoto S, Braid-Forbes MJ, Weber M, Berven SH. Readmission rates after decompression surgery in patients with lumbar spinal stenosis among Medicare beneficiaries. Spine (Phila Pa 1976). Apr 1 2013;38(7):591-596.

11. Schairer WW, Carrer A, Deviren V, et al. Hospital readmission after spine fusion for adult spinal deformity. Spine (Phila Pa 1976). Sep 1 2013;38(19):1681-1689.

12. Wang MC, Kreuter W, Wolfla CE, Maiman DJ, Deyo RA. Trends and variations in cervical spine surgery in the United States: Medicare beneficiaries, 1992 to 2005. Spine (Phila Pa 1976). Apr 20 2009;34(9):955-961; discussion 962-953.

\section{Disclosures}

The authors declare no relevant disclosures for this paper.

\section{Corresponding author}

Thomas Cheriyan, MD, 306 East, 15th Street, New York City, New

York 10003. thomascheriyan@gmail.com.

Published 26 May 2015.

This manuscript is generously published free of charge by ISASS, the International Society for the Advancement of Spine Surgery. Copyright @ 2015 ISASS. To see more or order reprints or permissions, see http://ijssurgery.com. 